\title{
IQ discrepancy differentiates levels of fine motor skills and their relationship in children with autism spectrum disorders
}

This article was published in the following Dove Press journal:

Neuropsychiatric Disease and Treatment

Tzu-Ying Yu'

Willy Chou ${ }^{2,3}$

Julie Chi Chow ${ }^{4}$

Chien-Ho Lin ${ }^{5}$

Li-Chen Tung ${ }^{2,6}$

Kuan-Lin Chen ${ }^{7,8}$

'Department of Occupational

Therapy, College of Medicine, I-Shou University, Kaohsiung, ${ }^{2}$ Department of Physical Medicine and Rehabilitation, Chi-Mei Medical Center, ${ }^{3}$ Department of Recreation and Health Care Management, Cha Nan University of Pharmacy and Science, ${ }^{4}$ Department of Pediatrics, ${ }^{5}$ Department of Psychiatry, Chi Mei Medical Center, Tainan, ${ }^{6}$ Department of Physical Medicine and Rehabilitation, Da Chien General Hospital, Miaoli, ${ }^{7}$ Department of Occupational Therapy, College of Medicine, National Cheng Kung University, ${ }^{8}$ Department of Physical Medicine and Rehabilitation, National Cheng Kung University Hospital, Tainan, Taiwan
Correspondence: Kuan-Lin Chen Department of Occupational Therapy, College of Medicine, National Cheng Kung University, No I University Road, Tainan City 70I, Taiwan

Tel +88662353535 ext 5906

Email klchen@mail.ncku.edu.tw
Purpose: We investigated 1) the impact of differences in intelligence quotient discrepancy (IQD) on motor skills of preschool-aged children with autism spectrum disorders (ASD); 2) the relationships between IQD and motor skills in preschool-aged children with ASD.

Methods: A total of 127 ASD preschool-aged children were divided into three groups according to the size of the IQD: IQD within 1 standard deviation (1SD; EVENIQ; $n=81$ ), discrepantly higher verbal intelligence quotient (VIQ; $\mathrm{n}=22$; VIQ $>$ performance intelligence quotient [PIQ] above 1SD [ $\geq 15$ points]), and discrepantly higher PIQ ( $n=24$; PIQ $>$ VIQ above 1SD [ $\geq 15$ points]). Children's IQD and motor skills were determined with the Wechsler Preschool and Primary Scale of Intelligence ${ }^{\mathrm{TM}}$ - Fourth Edition and the motor subtests of the Comprehensive Developmental Inventory for Infants and Toddlers (CDIIT), respectively.

Results: One-way analysis of variance revealed significant group differences for the fine motor domain of the CDIIT and the visual-motor coordination subtest $(F=3.37-4.38, p<0.05)$. Children with discrepantly higher PIQ were associated with better fine motor skills than were children with even IQD and those with discrepantly higher VIQ, and vice versa. IQD (PIQ VIQ) had significant positive correlations with the fine motor domain and fine motor subtests of the CDIIT $(r=0.18-0.29, p<0.05)$.

Conclusion: The IQD can identify different levels of fine motor skills in preschool-aged children with ASD. This study suggests important implications for clinicians, therapists, and researchers: discrepantly higher PIQ could be related to better visual-motor coordination, and discrepantly higher VIQ could be related to poor visual-motor coordination. Furthermore, the results support that when therapists are working with preschool-aged children with ASD who are developing fine motor skills or undertaking fine motor tasks related to visual-motor coordination, they may need to pay attention to the children's IQD.

Keywords: intelligence discrepancy, autistic disorder, motor development, child

\section{Introduction}

Autism spectrum disorders (ASD) are associated with highly varied cognitive profiles. ${ }^{1-4}$ There has been longstanding interest in the various intellectual abilities of individuals with ASD, such as intelligence quotient discrepancy (IQD). IQD refers to the discrepancy between verbal and nonverbal intellectual abilities, indicating an atypical pattern of verbal-performance IQ differences (ie, the distance between measured verbal intelligence quotient [VIQ] and performance intelligence quotient [PIQ]). ${ }^{5}$ Recent work suggests that an IQD may be a particular characteristic of the autism spectrum. Joseph, Tager-Flusberg, and Lord's study demonstrated a significantly higher rate of discrepancies between verbal and nonverbal ability scores in preschool-aged 
and school-aged children with ASD (ages 4-13) than in the normative sample. ${ }^{6}$ Therefore, explorations have focused on the associations of IQD with several aspects of neurological development of children with ASD, including executive function, ${ }^{7}$ social skills, communicative skills, and adaptive behaviors. ${ }^{6,8,9}$ For example, Black et $\mathrm{al}^{8}$ reported a possible association between the discrepantly higher VIQ or higher PIQ ( $\geq 11$ points) in 78 school-aged children with high-functioning ASD and their social difficulties and adaptive behaviors. A study by Joseph et $\mathrm{al}^{6}$ also found that 120 children with ASD, ages 4-13 years (mean Full Scale Intelligence Quotient [FSIQ] =76.7 measured by Differential Ability Scales) with significantly better ( $\geq 9$ points) PIQ than VIQ, had more social symptoms than children with either evenly developing VIQ and PIQ or better developed VIQ than PIQ. In addition to the aforementioned neurological developmental limitations of children with ASD, their motor skills may be compromised due to their IQD. ${ }^{10}$

The deficits of motor skills of children with ASD are widely reported in the literature. ${ }^{11-16}$ The Diagnostic and Statistical Manual of Mental Disorders fourth edition, text revision (DSM-IV-TR) ${ }^{17}$ cites "abnormalities of posture" as a feature of autistic disorder and "motor clumsiness" as a feature of Asperger disorder. Manjiviona and Prior $^{14}$ found that $50 \%$ of children with Asperger syndrome and $67 \%$ of children with autism exhibited motor dysfunction on the Henderson Test of Motor Impairment. ${ }^{18}$ Using the Bruininks-Oseretsky Test of Motor Proficiency, Ghaziuddin and Butler ${ }^{19}$ found that movement problems were common in children with Asperger syndrome, autism, or pervasive development disorder not otherwise specified. ${ }^{20}$ If a relationship between neurocognitive function and motor can be established in the ASD population, interventions may be developed to help reduce their motor deficit, as has been observed in several studies on brain damage ${ }^{21,22}$ and those in children who have sensorineural problems. ${ }^{23}$

As far as we know, the relationship between IQD and motor skills has not been tested in children with ASD, although motor skill development is related to a range of cognitive functions. ${ }^{24,25}$ A Wechsler ${ }^{5}$ IQ profile with VIQ depressed relative to PIQ and a peak subtest score on Block Design $^{26}$ has traditionally been associated with ASD, and VIQ has been suggested to have a possible connection to visuospatial ability. PIQ appears important to motor function for the reason that spatial relationships, sequencing memory, and visual perception and visual motor coordination embedded in PIQ are essential for children to perform motor activities. ${ }^{27,28}$ Moreover, there have been concerns about the relevance of motor development to the uneven intellectual abilities of individuals with ASD. The poor motor performance observed in children with borderline and mild intellectual delay has been suggested to be related to their impaired intellectual functioning. Piaget and Inhelder ${ }^{29}$ argued that cognitive development relies on motor functioning, and recent findings also suggest that motor performance and intelligence are linked. ${ }^{30,31}$ Although Kamphaus ${ }^{32}$ and Sattler ${ }^{33}$ hypothesized that IQD could be related to the development of visual-motor integration, they did not provide empirical evidence to support their postulations - that PIQ below VIQ may present limitations in visual-motor integration, and that VIQ below PIQ may present better visual-motor integration. ${ }^{32,33}$ A study by $\mathrm{Yu}$ et $\mathrm{al}^{34}$ found that VIQ $>$ PIQ discrepancy is negatively associated with motor competence in preschool-aged children at risk for developmental delays. They confirmed that preschool-aged children with discrepantly higher VIQ had worse motor competence in body-movement coordination and visual-motor integration. ${ }^{34}$ Therefore, when assessing motor delays or difficulties, clinicians and researchers need to consider children's cognitive function when determining whether their motor output is consistent with or below their intelligence and age. However, previous papers focused on the relationships between motor skills and general cognitive delay. The clinical value of IQD has been much less exhaustively investigated, especially in children with ASD.

To the best of our knowledge, motor skills development is related to a range of cognitive functions, including general intelligence or PIQ; however, the relevance of IQ to the etiology and neurological development of ASD remains unclear. Recent studies have suggested that an IQD may be a particular characteristic of ASD. In previous studies, Kamphaus $^{32}$ and Sattler ${ }^{33}$ hypothesized that IQD was related to visual-motor integration, but empirical evidence was lacking. Yu et $\mathrm{a}^{34}$ showed that IQD and motor development are linked in children with developmental delay. There have been concerns about the relevance of motor development to the uneven intellectual abilities of individuals with ASD. Examining IQD and motor skills and identifying whether IQD is a possible determinant for the motor performance of children with ASD can provide clinicians, educators, and therapists with crucial information concerning the developmental strengths and weaknesses in cognition and motor skills of these children, along with recommendations for possible remediation plans for their dysfunction.

Therefore, the current investigation seeks to explore the impact of IQD on motor skills in children with ASD. Based on the study by Yu et $\mathrm{al}^{134}$ confirming that IQD indicates levels of motor competence in preschool-aged children at risk for developmental delays, we hypothesized that IQD may be 
able to differentiate different levels of fine motor and gross motor skills in children with ASD. A discrepantly higher VIQ may indicate limitations in motor skills; on the other hand, a discrepantly higher PIQ may suggest better motor skills. In this study, we predefined groups of preschool-aged children with ASD, aged from 2.5 to 6 , according to the size of the IQD to test its association with motor skills. The goals of this present study were to investigate links between IQD and motor skills among preschool-aged children with ASD. In order to achieve these aims, we investigated 1) the impact of IQD on the motor skills of preschool-aged children with ASD and 2) the relationships between IQD and motor skills in preschool-aged children with ASD.

\section{Methods}

\section{Participants}

The participants were drawn from a retrospective chart review of 127 children for the period 2012-2014 from the Child Development and Assessment Center of Chi Mei Medical Center in Taiwan. Written informed consent was obtained from all participants and their parents or legal guardians in accordance with study protocols and procedures approved by the Institutional Review Board of Chi Mei Medical Center. All participants met the following inclusion criteria: 1) age 2.5-6 years and 2) clinical referral with an autism spectrum diagnosis evaluated by a multidisciplinary team at the Child Development and Assessment Center in Taiwan. All participants met the diagnostic criteria for Autistic disorder, Asperger's disorder, or Pervasive Developmental Disorders - Not Otherwise Specified (PDD-NOS) according to the DSM-IV-TR, ${ }^{17}$ or of autism spectrum disorder (ASD) according to the fifth edition of the Diagnostic and Statistical Manual of Mental Disorders (DSM-V) ${ }^{35}$ by experienced child psychiatrists and psychologists who evaluated them over several visits. The participants included 127 children with ASD (105 boys, 22 girls), from 30 to 71 months in age (mean age 51.05 months; standard deviation $[\mathrm{SD}]=10.49$ months). Of these 127 children, most (89.7\%) had normal birth weights of more than $2,500 \mathrm{~g}$. The average age of the parents of the children was 35.88 (range of 23-56) years. Over $86 \%$ of the children's mothers had finished senior high school or earned a higher degree. Table 1 shows the demographic characteristics of all the participants.

\section{Instrumentation}

\section{The Comprehensive Developmental Inventory for Infants and Toddlers (CDIIT)}

The motor subtests of the Comprehensive Developmental Inventory for Infants and Toddlers (CDIIT) were used for
Table I Demographic characteristics of all participants $(n=127)$

\begin{tabular}{|c|c|}
\hline Characteristics & Frequency \\
\hline Age, months, mean (SD/range) & $5 I .05(|0.49 / 30-7|)$ \\
\hline Gender, male/female, n (\%) & $105 / 22(83 / 17)$ \\
\hline \multicolumn{2}{|l|}{ Birth weight (g), n (\%) } \\
\hline Normal birth weight $(\geq 2,500)$ & II 4 (89.7) \\
\hline Low birth weight $(1,500$ to $<2,500)$ & II (8.7) \\
\hline Very low birth weight $(<I, 500)$ & $2(1.6)$ \\
\hline Age of the fathers in years, mean (SD/range) & $37.55(5.78 / 25-56)$ \\
\hline Age of the mothers in years, mean (SD/range) & $34.20(4.76 / 23-47)$ \\
\hline \multicolumn{2}{|l|}{ Education level of the mothers, $\mathrm{n}(\%)$} \\
\hline$\geq 17$ years (postgraduate) & $7(5.8)$ \\
\hline 13-16 years (some college or technical school) & $56(46.3)$ \\
\hline $10-12$ years (senior high school) & $42(34.7)$ \\
\hline 7-9 years (junior-high school) & II (9.1) \\
\hline 6 years (elementary-school graduate) & $5(4.1)$ \\
\hline
\end{tabular}

measuring the motor performance of the children participating in this study. The CDIIT, a standardized and norm-referenced measurement for assessing a child's developmental level for children aged 3-72 months, is commonly used in Taiwan for developmental diagnosis. ${ }^{36,37}$ The CDIIT consists of five subtests assessing performance in five domains: cognitive (81 items), motor (97 items), language (62 items), self-help (47 items), and social development (56 items). ${ }^{37}$ The motor subtest (motor domain) has two composites: the gross motor skill (CDIIT-GM) and the fine motor skill (CDIIT-FM) domains. The CDIIT-GM includes the gravity compensation, locomotion, and body-movement coordination subtests. The CDIIT-FM includes the basic hand use and visual-motor coordination subtests. ${ }^{37}$

Each CDIIT item is dichotomous, where 0 indicates fail and 1 indicates pass, either during the test or according to the observations of the caregiver. The raw scores of the CDIIT can be transformed into developmental quotients (DQs) to classify children as delayed (DQ $<70$ ), borderline (70 $\leq \mathrm{DQ}<85$ ), or normal ( $\mathrm{DQ} \geq 85$ ). The CDIIT provides DQs for the aforementioned five domains, 19 subdomains, and the whole test, with a mean score of 100 and an SD of $15 .{ }^{37}$ The CDIIT has acceptable reliability and validity, ${ }^{36-40}$ and has shown acceptable test-retest reliability (intraclass correlation coefficient $[\mathrm{ICC}]=0.76-1.00, p<0.01$ ), inter-rater reliability (ICC $=0.76-1.00, p<0.01),{ }^{39}$ and internal consistency (Cronbach's $\alpha=0.75-0.99) .{ }^{37}$ Furthermore, its validity for decisions has been established. ${ }^{36,37,39,41-43}$ In terms of the validity of the CDIIT, the content validity, ${ }^{37}$ concurrent validity with the Bayley Scale of Infant Development second edition, ${ }^{36,40}$ predictive validity for special education status ${ }^{41}$ and construct validity of the CDIIT $^{38}$ have all been determined. 


\section{Wechsler Preschool and Primary Scale of Intelligence ${ }^{\mathrm{TM}}$ - Fourth Edition}

We used the Wechsler Preschool and Primary Scale of Intelligence ${ }^{\mathrm{TM}}$ - Fourth Edition (WPPSI ${ }^{\mathrm{TM}}-\mathrm{IV}$ ) in the study to evaluate the children's intelligence and generate three summary scores: FSIQ, which represents general intellectual functioning; VIQ, which represents verbal reasoning and comprehension; and PIQ, which represents fluid reasoning, spatial processing, and visual-motor integration. ${ }^{5}$ The WPPSI ${ }^{\text {TM}}$-IV is an individually administered intelligence tool that assesses cognitive functioning among children aged 2 years and 6 months through 7 years and 7 months. Younger children are assessed with fewer subtests than are older children.

The VIQ test primarily assesses conceptual and logical reasoning, vocabulary, and information through the verbal mode. Questions are asked verbally by the rater and answered verbally by the child. The PIQ test evaluates spatial relations, visual sequencing, and other visual perceptual and visual motor skills, largely in a nonverbal format. Together, the VIQ and PIQ give the FSIQ for presenting an overall measure of general intelligence. An average IQ on the psychometric curve is demarcated as an FSIQ of 100. Standard deviations are significant at $15(p<0.01)$, and denote mild intellectual disability at 55-70, borderline intelligence at 70-85, normal intelligence at 85 or above, above average intelligence at 115-129, and superior intelligence at 130 or above.

The WPPSI ${ }^{\text {TM}}-I V$ has been shown to be highly reliable in terms of interrater reliability $(0.84-0.94)$, test-retest reliability (0.86-0.91), and split-half reliability (0.77-0.87). ${ }^{5,44}$ Moreover, the WPPSI ${ }^{\mathrm{TM}}$-IV shows good validity with studies related to concurrent validity with the StanfordBinet, Wechsler Intelligence Scale for Children (WISC), and WISC-R, predictive validity to achievement test scores, and construct validity based on factor analytic studies. ${ }^{44,45}$

\section{Statistical analyses}

To begin, all the participating children were classified into three subgroups according to the size of the IQD. IQD is defined as the absolute point value of the difference between VIQ and PIQ. ${ }^{46}$ When the discrepancy is above 15 points (ie, a 15-point discrepancy is required at the $1 \%$ level of significance), ${ }^{44,47}$ IQD is used to determine statistical differences in typical populations, or clinical significance. Therefore, three subgroups were identified on the basis of the size of the IQD, including 1) EVEN IQ, or equivalent IQ, containing those with a discrepancy within 1SD ( $<15$ points, indicating no significant IQD); 2) discrepantly higher VIQ; and 3) discrepantly higher PIQ, containing those with a discrepancy of more than $1 \mathrm{SD}$ ( $\geq 15$ points, indicating a significant IQD) to examine the impacts that different IQDs have on motor skills. A discrepantly higher VIQ means that the PIQ score is significantly below the VIQ score (by more than 15 points); a discrepantly higher PIQ means that the VIQ score is significantly below the PIQ score (by $>15$ points).

Next, we analyzed the descriptive statistics for important variables including age, gender, birth weight, ages of the parents, education level of the mothers, FSIQ, VIQ, PIQ, IQD, and subtest scores of the CDIIT from all of the participating children. In addition, we tested the differences in basic clinical characteristics (ie, age, gender, weight of birth, mother's education, and parents' ages) within the three subgroups with the one-way analysis-of-variance (ANOVA) $F$-test for continuous variables, and with overall chi-square tests for dichotomous variables.

In addition, to check for statistical differences among the three subgroups, an ANOVA was conducted after testing the homoscedasticity with Levene's test. ${ }^{48}$ When significant differences were identified, one-way ANOVAs with post hoc analysis - based on Bonferroni post hoc comparisons ${ }^{48}$ were conducted to account for differences in the DQ of the CDIIT-GM and CDIIT-FM between the subgroups. Finally, in order to examine the relationships between the IQD and both CDIIT-GM and CDIIT-FM, Pearson correlation coefficients $(r)^{48}$ were used. Only $p$-values $<0.05$ were considered statistically significant for all tests. $\operatorname{SPSS}^{49}$ v.18 was used for all analyses.

\section{Results}

\section{Descriptive statistics}

A total sample of 127 participating children - of which 81 children were in the EVENIQ group, 22 children were in the discrepantly higher VIQ group, and 24 children were in the discrepantly higher PIQ group - were included in this study. Descriptive data for the three subgroups are shown in Table 2. There were no significant group differences in age $\left(F_{(2,124)}=2.58, p=0.08\right)$, gender $(p=0.39$, Pearson chi-square test), birth weight $\left(F_{(2,110)}=0.48, p=0.62\right)$, father's $\left(F_{(2,17)}=0.17\right.$, $p=0.84)$ and mother's ages $\left(F_{(2,120)}=1.65, p=0.20\right)$, or mother's education $\left(F_{(2,118)}=1.44, p=0.24\right)$. These results indicated no significant between-group differences with regard to the clinical characteristics. The FSIQs of the total sample were 42-131, with an average of 76.43 ( $S D=15.40$, with a wide range of intelligence) that was below the FSIQ of 100 in the psychometric curve. 
Table 2 Comparison among the three ASD subgroups (EVENIQ group, discrepantly higher VIQ, and discrepantly higher PIQ) on age, FSIQ, VIQ, PIQ, and motor subtests of the CDIIT

\begin{tabular}{|c|c|c|c|c|c|c|c|c|c|c|c|c|c|c|}
\hline & \multicolumn{3}{|c|}{$\begin{array}{l}\text { All participants } \\
(n=127)\end{array}$} & \multicolumn{3}{|c|}{$\begin{array}{l}\text { EVENIQ } \\
(n=8 I)\end{array}$} & \multicolumn{3}{|c|}{$\begin{array}{l}\text { Discrepantly higher } \\
\text { VIQ }(n=22)\end{array}$} & \multicolumn{3}{|c|}{$\begin{array}{l}\text { Discrepantly higher } \\
\text { PIQ }(n=24)\end{array}$} & \multicolumn{2}{|c|}{$\begin{array}{l}\text { Group } \\
\text { differences } \\
\text { among EVENIQ, } \\
\text { discrepantly } \\
\text { higher VIQ and } \\
\text { discrepantly } \\
\text { higher PIQ }\end{array}$} \\
\hline & Mean & SD & Range & Mean & SD & Range & Mean & SD & Range & Mean & SD & Range & $\boldsymbol{F}$ & $p$-value \\
\hline Age (month) & 51.05 & 10.49 & $30-7 \mid$ & 50.22 & 11.01 & $30-70$ & 49.41 & 9.06 & $36-67$ & 55.33 & 9.05 & 33-71 & 2.58 & 0.08 \\
\hline FSIQ & 76.43 & 15.40 & $42-131$ & 75.41 & 17.26 & $42-13 \mid$ & 73.45 & 13.25 & $54-106$ & 80.58 & 7.22 & $68-101$ & 2.56 & 0.13 \\
\hline VIQ & 77.42 & 15.11 & $46-131$ & 76.63 & 16.17 & $46-13 \mid$ & 86.27 & 13.80 & $65-114$ & 71.96 & 7.68 & $59-89$ & 5.88 & $0.004 *$ \\
\hline PIQ & 79.50 & 17.94 & $46-123$ & 77.33 & 16.00 & $46-123$ & 65.45 & 12.80 & $47-94$ & 99.67 & 9.65 & $78-117$ & 34.44 & $<0.000 I^{*}$ \\
\hline IQD (absolute value) & 13.12 & 10.45 & $0-52$ & 6.70 & 3.69 & $0-14$ & 20.82 & 6.76 & $15-44$ & 27.71 & 9.26 & $16-52$ & 150.27 & $<0.000 I^{*}$ \\
\hline CDIIT-GM (DQs) & 79.32 & 13.11 & $54-118$ & 87.40 & 13.12 & $54-100$ & 76.91 & 11.85 & 54-101 & 80.80 & 10.30 & $54-98$ & 0.35 & 0.72 \\
\hline Gravity compensation & 100 & 0 & 100 & 100 & 0 & 100 & 100 & 0 & 100 & 100 & 0 & 100 & NA & NA \\
\hline Locomotion & 84.23 & 16.31 & $54-115$ & 84.09 & 17.40 & $54-115$ & 81.95 & 14.11 & $54-109$ & 86.79 & 14.55 & $67-109$ & 0.56 & 0.60 \\
\hline Body-movement coordination & 81.06 & 12.16 & $54-109$ & 81.30 & 12.95 & $54-109$ & 79.18 & 11.79 & $54-101$ & 81.96 & 9.80 & $60-103$ & 0.34 & 0.72 \\
\hline CDIIT-FM (DQs) & 76.54 & 15.16 & $54-112$ & 75.70 & 15.60 & $54-112$ & 71.14 & 12.36 & 54-92 & 84.61 & 13.27 & $65-112$ & 5.10 & $0.008^{*}$ \\
\hline Basic hand use & 83.60 & 16.33 & $54-108$ & 82.09 & 16.63 & $54-108$ & 81.91 & 15.60 & 54-107 & 90.48 & 14.72 & $54-107$ & 2.57 & 0.08 \\
\hline Visual-motor coordination & 78.65 & 14.73 & $54-118$ & 77.66 & 14.79 & $54-112$ & 73.55 & 11.53 & 54-92 & 86.91 & 14.49 & $67-118$ & 5.50 & $0.005^{*}$ \\
\hline
\end{tabular}

Notes: Discrepantly higher VIQ group refers to VIQ>PIQ above ISD; discrepantly higher PIQ group refers to PIQ $>$ VIQ above ISD; ISD =I5 points discrepancy. EVENIQ group refers to IQD within ISD. $* p<0.01$.

Abbreviations: ASD, autism spectrum disorders; CDIIT, The Comprehensive Developmental Inventory for Infants and Toddlers; DQs, developmental quotients; FSIQ, Full Scale Intelligence Quotient; IQD, intelligence quotient discrepancy; NA, not applicable; PIQ, performance intelligence quotient; VIQ, verbal intelligence quotient; WPPSI TM _IV, Wechsler Preschool and Primary Scale of Intelligence ${ }^{\mathrm{TM}}$ - Fourth Edition; ISD, one standard deviation.

The total sample achieved the maximum score on the gravity compensation subtest of the CDIIT due to the fact that the subtest comprised items related to sitting and standing balance, which posed no challenge to our sample of ASD children. Because of the ceiling effect in the gravity compensation subtest, we excluded the subtest from the following analyses.

\section{Group differences in the motor subtests of the CDIIT}

Variables of the study met the assumption of equal variances on Levene's test; therefore, one-way ANOVAs were then conducted. The results of the ANOVAs revealed statistically significant differences among the three subgroups $(p<0.05)$ for DQ of the CDIIT-FM domain $(F=5.10, p=0.008)$ and visual-motor coordination subtest of the CDIIT $(F=5.50$, $p=0.005$; Table 2).

Subsequently, we employed post hoc Bonferroni tests to compare group differences in the CDIIT with statistical significance (Table 3). The scores of all subtests of the CDIIT for the children with discrepantly higher PIQ were higher than those of both the children with EVENIQ and those with discrepantly higher VIQ, but the differences were statistically significant only in the CDIIT-FM domain
Table 3 Analysis with Bonferroni post hoc comparisons between subgroups including EVENIQ $(n=8 \mathrm{I})$, discrepantly higher VIQ $(n=22)$, and discrepantly higher PIQ $(n=24)$

\begin{tabular}{|c|c|c|c|c|c|}
\hline $\begin{array}{l}\text { DQs of the } \\
\text { CDIIT }\end{array}$ & Group $^{a}$ & $\begin{array}{l}\text { Compared } \\
\text { group }^{\mathrm{a}}\end{array}$ & $\begin{array}{l}\text { Group mean } \\
\text { difference }\end{array}$ & SE & $p$-value \\
\hline \multirow[t]{3}{*}{ CDIIT-GM } & $\mathrm{PIQ}>\mathrm{VIQ}$ & EVENIQ & -1.49 & 2.99 & 1.0 \\
\hline & & $\mathrm{VIQ}>\mathrm{PIQ}$ & 3.05 & 3.67 & 1.0 \\
\hline & EVENIQ & $\mathrm{VIQ}>\mathrm{PIQ}$ & 1.49 & 3.13 & 1.0 \\
\hline \multirow[t]{3}{*}{ Locomotion } & $\mathrm{PIQ}>\mathrm{VIQ}$ & EVENIQ & 2.71 & 3.81 & 1.0 \\
\hline & & VIQ $>$ PIQ & 4.84 & 4.83 & 0.96 \\
\hline & EVENIQ & VIQ >PIQ & 2.13 & 3.94 & 1.0 \\
\hline Body-movement & $\mathrm{PIQ}>\mathrm{VIQ}$ & EVENIQ & 0.66 & 2.84 & 1.0 \\
\hline \multirow[t]{2}{*}{ coordination } & & VIQ >PIQ & 2.78 & 3.61 & 1.0 \\
\hline & EVENIQ & VIQ $>$ PIQ & 2.11 & 2.94 & 1.0 \\
\hline \multirow[t]{3}{*}{ CDIIT-FM } & $\mathrm{PIQ}>\mathrm{VIQ}$ & EVENIQ & 8.91 & 3.47 & $0.03^{*}$ \\
\hline & & VIQ >PIQ & 13.47 & 4.38 & $0.008 * *$ \\
\hline & EVENIQ & VIQ >PIQ & 4.56 & 3.54 & 0.60 \\
\hline \multirow[t]{3}{*}{ Basic hand use } & $\mathrm{PIQ}>\mathrm{VIQ}$ & EVENIQ & 8.39 & 3.82 & 0.08 \\
\hline & & $\mathrm{VIQ}>\mathrm{PIQ}$ & 8.57 & 4.81 & 0.23 \\
\hline & EVENIQ & $\mathrm{VIQ}>\mathrm{PIQ}$ & 0.18 & 3.88 & 1.0 \\
\hline Visual-motor & $\mathrm{PIQ}>\mathrm{VIQ}$ & EVENIQ & 9.25 & 3.37 & $0.02 *$ \\
\hline \multirow[t]{2}{*}{ coordination } & & VIQ >PIQ & 13.37 & 4.24 & $0.006 * *$ \\
\hline & EVENIQ & $\mathrm{VIQ}>\mathrm{PIQ}$ & 4.11 & 3.43 & 0.70 \\
\hline
\end{tabular}

Notes: ${ }^{a} E V E N I Q$ group refers to IQD within ISD; discrepantly higher VIQ group refers to $\mathrm{VIQ}>\mathrm{PIQ}$ of more than ISD; discrepantly higher PIQ group refers to $\mathrm{PIQ}>\mathrm{VIQ}$ of more than ISD; ISD $=15$ points discrepancy. ${ }^{*} p<0.05 ;{ }^{* *} p<0.0$ I.

Abbreviations: CDIIT, The Comprehensive Developmental Inventory for Infants and Toddlers; DQs, developmental quotients; SE, standard error; IQD, intelligence quotient discrepancy; PIQ, performance intelligence quotient; VIQ, verbal intelligence quotient; ISD, one standard deviation. 
( $F=3.47, p=0.03$ with the EVENIQ group; $F=4.38, p=0.08$ with the discrepantly higher VIQ group), and in the visualmotor coordination subtest ( $F=3.37, p=0.02$ for the EVENIQ group; $F=4.24, p=0.006$ for the discrepantly higher VIQ group). The scores of all motor subtests of the CDIIT of the EVENIQ group were higher than those of the discrepantly higher VIQ group; however, the differences were not statistically significant.

\section{Correlations between IQD and motor skills}

The results of the correlation between IQ profiles (including IQD, VIQ, and PIQ) and motor skills for the total sample are shown in Table 4. VIQ $(r=0.23-0.41, p<0.01)$ and PIQ ( $r=0.26-0.60, p<0.01)$, both, had significantly positive correlations with every subtest. The IQD (PIQ - VIQ) had significantly positive correlations with the CDIIT-FM ( $r=0.28, p=0.002)$, the basic hand use subtest ( $r=0.18, p=0.05$ ), and the visual-motor coordination subtest $(r=0.29, p=0.001)$.

A scatterplot was used to further examine the relationships between IQD (PIQ - VIQ) and the fine motor subdomain and visual-motor coordination subtest of the CDIIT by calculating regression lines for all participants. Figures 1 and 2 show the regression lines indicating that discrepantly higher PIQ was associated with better fine motor coordination and visual motor coordination.

\section{Discussion}

The current study investigated whether children with different levels of IQD have differences in motor skills and whether associations exist between IQD and motor skills in preschool-aged children with ASD. We analyzed the data in two ways: by comparing groups of children with discrepantly

Table 4 Associations between IQ profiles (ie, VIQ, PIQ, and IQD) and motor subtests of CDIIT in all participants

\begin{tabular}{llll}
\hline DQs of the CDIIT & \multicolumn{3}{l}{ All participants (n=I27) } \\
\cline { 2 - 4 } & IQD (PIQ - VIQ) & VIQ & PIQ \\
\hline CDIIT-GM & 0.09 & $0.30^{* *}$ & $0.33^{* *}$ \\
Locomotion & 0.09 & $0.34^{* *}$ & $0.37^{* *}$ \\
Body-movement coordination & 0.07 & $0.23^{* *}$ & $0.26^{* *}$ \\
CDIIT-FM & $0.28^{* *}$ & $0.41^{* *}$ & $0.60^{* *}$ \\
Basic hand use & $0.18^{*}$ & $0.25^{* *}$ & $0.37^{* *}$ \\
Visual-motor coordination & $0.29^{* *}$ & $0.40^{* *}$ & $0.60^{* *}$ \\
\hline
\end{tabular}

Notes: ${ }^{a} \mathrm{QQD}(\mathrm{PIQ}-\mathrm{VIQ})=$ Intelligence quotient discrepancy with value of the difference between PIQ and VIQ. ${ }^{*} p<0.05$; ${ }^{*} p<<0.01$.

Abbreviations: CDIIT, The Comprehensive Developmental Inventory for Infants and Toddlers test; CDIIT-GM, gross motor skill subscale of the Comprehensive Developmental Inventory for Infants and Toddlers; CDIIT-FM, fine motor skill subscale of the Comprehensive Developmental Inventory for Infants and Toddlers; $D Q$ s, developmental quotients; IQ, intelligence quotient; IQD, intelligence quotient discrepancy; PIQ, performance intelligence quotient; VIQ, verbal intelligence quotient.

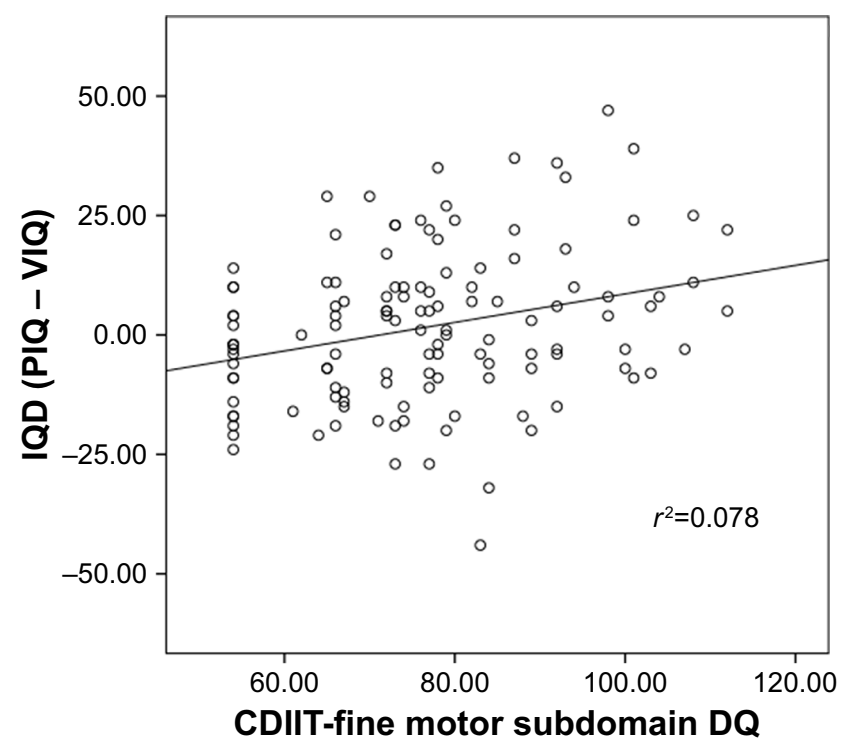

Figure I Scatterplot showing the relationship between IQD (PIQ - VIQ) and fine motor subdomain of CDIIT ( $n=127)$.

Abbreviations: CDIIT, The Comprehensive Developmental Inventory for Infants and Toddlers; DQ, developmental quotient; IQD, intelligence quotient discrepancy; $\mathrm{PIQ}$, performance intelligence quotient; VIQ, verbal intelligence quotient.

higher VIQ, EVENIQ, and discrepantly higher PIQ; as well as by using the full sample to examine correlations of the IQ indices (IQD, VIQ, and PIQ) and motor skills. Using both methods, we developed two essential findings. First, IQD can differentiate different levels of motor skills in fine motor tasks. Children with discrepantly higher PIQ greater than 1SD performed significantly better on fine motor skills, compared to children with even IQD and children with discrepantly higher VIQ greater than 1SD. Children with discrepantly

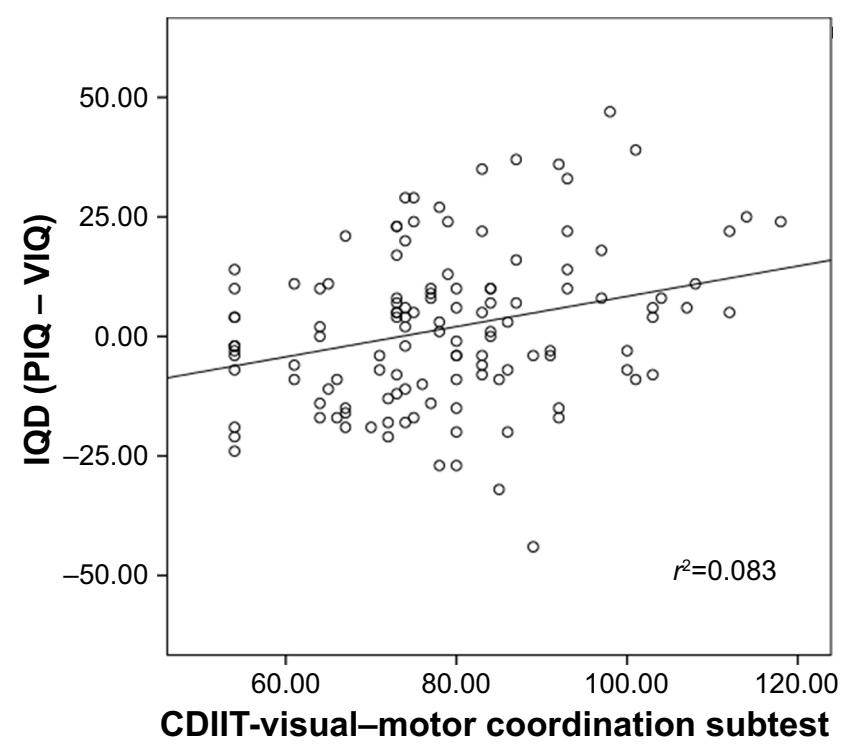

Figure 2 Scatterplot showing the relationship between IQD (PIQ - VIQ) and the visual-motor coordination subtest of the CDIIT ( $n=127)$.

Abbreviations: CDIIT, The Comprehensive Developmental Inventory for Infants and Toddlers; IQD, intelligence quotient discrepancy; PIQ, performance intelligence quotient; VIQ, verbal intelligence quotient. 
higher VIQ performed significantly worse on fine motor skills than did children with discrepantly higher PIQ. Second, we found discrepantly higher PIQ to be associated with greater fine motor skills, and discrepantly higher VIQ to be associated with poor fine motor skills in children with ASD. The results of this study provide evidence for clinicians, educators, and therapists that, in children with ASD, PIQ $>$ VIQ discrepancy could be related to better visual-motor coordination and VIQ $>$ PIQ discrepancy could be related to poor visual-motor coordination.

With respect to the significant group differences in fine motor skills among children with different levels of IQD, we concluded that the children with ASD performed differently on the fine motor tasks. This finding indicates that discrepantly higher PIQ may be strongly related to the development of fine motor skills and visual-motor coordination in preschool-aged children with ASD. Our discovery verifies the proposition by previous researchers ${ }^{32-34}$ that children with PIQ $>$ VIQ discrepancy possess better visual-motor integration. We further verified that discrepantly higher VIQ reveals limitations in a child's visual-motor integration, whereas discrepantly higher PIQ implies better motor development in visual-motor integration. Motor skills tend to rely on performance intelligence. When children have discrepantly higher VIQ, more attention should be paid to their fine motor development in terms of visual-motor integration.

The IQD was correlated with the CDIIT-FM domain, hand-use subtest, and visual-motor coordination subtest, but not correlated with the CDIIT-GM domain, suggesting that IQD is uniquely related to fine motor development in terms of visual-motor coordination in preschoolers with ASD, but is not related to gross motor deficit. A possible explanation for the association between IQD and motor ability is that motor skill deficits, although not pervasive, may be more apparent in activities demanding complex or interceptive actions in children with ASD. Whyatt and Craig ${ }^{10}$ examined the motor development in children with ASD aged 7-10 years and found significant motor impairment in ball skills, manual dexterity, and balance ability in these children. If, instead of the general motor tasks in the CDIIT, the children were asked to execute more complicated or difficult motor activities, such as physical exercises, ball games, or dancing, then children with discrepantly higher PIQ might show significantly better motor skills than might the other two groups because of their higher PIQ. Our findings are consistent with those of previous studies that provide evidence for specific areas of motor impairment in ASD children, such as manual dexterity ${ }^{15,50-54}$ and ball skills, ${ }^{14,15,50,52}$ as compared to typically developing children. Based on the general recommendation that a child's motor skills should be assessed from diverse perspectives with multiple motor assessments, further research is needed to explore the links between IQD and advanced gross and fine motor skills by using measures with more difficult or complex motor tasks. Furthermore, future studies should investigate what level of motor performance can be expected, given a child's measured IQD.

With respect to the second finding, that the IQD with higher PIQ was positively correlated with the CDIIT-FM and visual-motor coordination subtest, this result manifested that, as the IQD increases, the scores of the CDIIT-FM may increase or decrease. When children present a larger VIQ $>$ PIQ discrepancy, they perform worse on fine motor tasks, and vice versa. These findings were consistent with the results of between-group comparisons in this study, demonstrating that VIQ $>$ PIQ discrepancy and PIQ $>$ VIQ discrepancy have a specific relationship with fine motor skills, which is more obvious in children whose IQD is greater than $1 \mathrm{SD}$.

\section{Limitations}

The results of this study need to be interpreted with caution because of certain known limitations. First of all, this retrospective study was intended to explore the relationships between IQD and motor competence in a sample of children with ASD; thus, it did not include a control group of typically developing children. A control population consisting of typically developing children with and without IQD is suggested for future studies to compare ASD and typically developing children in their measured IQD and motor skills. Second, a larger sample size of children with IQD is suggested for future studies to investigate the relevance of IQD and motor performance in ASD children. The most significant group differences on the motor subtests of the CDIIT emerged from the groups of discrepantly higher PIQ and discrepantly higher VIQ, which comprised comparably small sample sizes of 22-24. Increasing the sample size in the future will provide more valid interpretation of the findings. In addition, a recommendation for a larger sample size of children with significant IQD and children with superior intellectual function could increase the understanding of the preliminary findings reported in this study. Third, although this pivotal study has provided evidence on how motor subskills are linked to IQD, the subtest gravity compensation was not appropriate for use in our participants because the items were not even slightly challenging for the participants. Other comprehensive motor-focused assessment tools may lead to specific insight about how these motor skills emerge and develop in preschool-aged children with ASD. Fourth, while the findings support an association between IQD and 
visual-motor coordination, they cannot be interpreted to suggest causality.

\section{Conclusion}

Although cognition and motor skills do not always covary, they do in most children with ASD. This study provided a noteworthy discovery: preschool-aged children with ASD with different types of IQD have differences in motor performance. Especially, children with discrepantly higher PIQ exhibit better visual-motor integration and fine motor coordination than do children without significant IQD and children with discrepantly higher VIQ. Moreover, children with discrepantly higher VIQ are likely to have fine motor limitations. It is important to remember that dysfunction in one component of the neural system will affect the other components, particularly in a developing brain. The results of this study should encourage clinicians, educators, and therapists to take IQD into consideration when measuring children's fine motor function and to analyze the patterns of motor skills in preschool-aged children with ASD. Possible fine motor problems of children with ASD may be detected early based on routine evaluations of their cognitive profiles. Furthermore, the fine motor coordination impairment can be explained in terms of IQD. When therapists are working with preschoolers with ASD who are developing fine motor skills or performing fine motor tasks related to visual-motor coordination, they may need to pay attention to the children's IQD.

\section{Disclosure}

The authors report no conflicts of interest in this work.

\section{References}

1. de Bruin EI, Verheij F, Ferdinand RF. WISC-R subtest but no overall VIQ-PIQ difference in Dutch children with PDD-NOS. J Abnorm Child Psychol. 2006;34(2):263-271.

2. Ghaziuddin M, Mountain-Kimchi K. Defining the intellectual profile of Asperger Syndrome: comparison with high-functioning autism. J Autism Dev Disord. 2004;34(3):279-284.

3. Lai MC, Lombardo MV, Ruigrok AN, et al; MRC AIMS Consortium. Cognition in males and females with autism: Similarities and differences. PLoS One. 2012;7(10):e47198.

4. Noterdaeme M, Wriedt E, Höhne C. Asperger's syndrome and highfunctioning autism: language, motor and cognitive profiles. Eur Child Adolesc Psychiatry. 2010;19(6):475-481.

5. Wechsler D. Wechsler Intelligence Scale for Children. 4th ed. San Antonio, TX: Psychological Corporation; 2003.

6. Joseph RM, Tager-Flusberg H, Lord C. Cognitive profiles and socialcommunicative functioning in children with autism spectrum disorder. J Child Psychol Psychiatry. 2002;43(6):807-821.

7. Kalbfleisch ML, Loughan AR. Impact of IQ discrepancy on executive function in high-functioning autism: insight into twice exceptionality. J Autism Dev Disord. 2012;42(3):390-400.

8. Black DO, Wallace GL, Sokoloff JL, Kenworthy L. Brief report: IQ split predicts social symptoms and communication abilities in highfunctioning children with autism spectrum disorders. $J$ Autism Dev Disord. 2009;39(11):1613-1619.
9. Klin A, Saulnier CA, Sparrow SS, Cicchetti DV, Volkmar FR, Lord C. Social and communication abilities and disabilities in higher functioning individuals with autism spectrum disorders: the Vineland and the ADOS. J Autism Dev Disord. 2007;37(4):748-759.

10. Whyatt CP, Craig CM. Motor skills in children aged 7-10 years, diagnosed with autism spectrum disorder. J Autism Dev Disord. 2012;42(9): 1799-1809.

11. Berkeley SL, Zittel LL, Pitney LV, Nichols SE. Locomotor and object control skills of children diagnosed with autism. Adapt Phys Activ $Q$. 2001;18(4):405-416.

12. Beversdorf DQ, Anderson JM, Manning SE, et al. Brief report: Macrographia in high-functioning adults with autism spectrum disorder. J Autism Dev Disord. 2001;31(1):97-101.

13. Ghaziuddin M, Butler E, Tsai L, Ghaziuddin N. Is clumsiness a marker for Asperger syndrome? J Intellect Disabil Res. 1994;38(Pt 5): 519-527.

14. Manjiviona J, Prior M. Comparison of Asperger syndrome and highfunctioning autistic children on a test of motor impairment. J Autism Dev Disord. 1995;25(1):23-39.

15. Miyahara M, Tsujii M, Hori M, Nakanishi K, Kageyama H, Sugiyama T. Brief report: motor incoordination in children with Asperger syndrome and learning disabilities. J Autism Dev Disord. 1997;27(5): 595-603.

16. Rinehart NJ, Bradshaw JL, Brereton AV, Tonge BJ. Movement preparation in high-functioning autism and Asperger disorder: a serial choice reaction time task involving motor reprogramming. $J$ Autism Dev Disord. 2001;31(1):79-88.

17. American Psychiatric Association. Diagnosis and Statistical Manual of Mental Disorder. 4th ed. Washington, DC: American Psychiatric Association Press; 2000.

18. Stott DH, Moyes FA, Henderson SE. The Test of Motor Impairment. San Antonio, TX: The Psychological Corporation; 1972.

19. Ghaziuddin M, Butler E. Clumsiness in autism and Asperger syndrome: a further report. J Intellect Disabil Res. 1998;42(Pt 1):43-48.

20. Bmininks RH. Bruininks-Oseretsky Test of Motor Proficiency: Examiner's manual. Circle Pines, MN: American Guidance Service; 1978.

21. Hadders-Algra M. Early brain damage and the development of motor behavior in children: clues for therapeutic intervention? Neural Plast. 2001;8(1-2):31-49.

22. Virji-Babul N, Kerns K, Zhou E, Kapur A, Shiffrar M. Perceptual-motor deficits in children with Down syndrome: Implications for intervention. Downs Syndr Res Pract. 2006;10(2):74-82.

23. Rine RM, Braswell J, Fisher D, Joyce K, Kalar K, Shaffer M. Improvement of motor development and postural control following intervention in children with sensorineural hearing loss and vestibular impairment. Int J Pediatr Otorhinolaryngol. 2004;68(9):1141-1148.

24. Campos JJ, Anderson DI, Barbu-Roth MA, Hubbard EM, Hertenstein MJ, Witherington D. Travel broadens the mind. Infancy. 2000;1(2): 149-219.

25. Piek JP, Dawson L, Smith LM, Gasson N. The role of early fine and gross motor development on later motor and cognitive ability. Hum Mov Sci. 2008;27(5):668-681.

26. Lincoln AJ, Allen M, Kilman A. The assessment and interpretation of intellectual abilities in people with autism. In: Schopler E, Mesibov G, editors. Learning and Cognition in Autism. New York: Plenum; 1995: 89-117.

27. Carroll JB. Human Cognitive Abilities: A Survey of Factor-Analytic Studies. Cambridge, England: Cambridge University Press; 1993.

28. Carroll JB. The three-stratum theory of cognitive abilities. In: Flanagan DP, Genshaft JL, Harrison PL, editors. Contemporary Intellectual Assessment: Theories, Tests, and Issues. NewYork: Guilford Press; 1997: $122-130$.

29. Piaget J, Inhelder B. The Psychology of the Child. New York: Basic Books; 1966.

30. Smits-Engelsman B, Hill EL. The relationship between motor coordination and intelligence across the IQ range. Pediatrics. 2012;13(4): e950-e956. 
31. Wassenberg R, Feron FJ, Kessels AG, et al. Relation between cognitive and motor performance in 5- to 6-year-old children: results from a large-scale cross-sectional study. Child Dev. 2005;76(5):1092-1103.

32. Kamphaus RW. Clinical Assessment of Children's Intelligence: A Handbook for Professional Practice. Boston, MA: Allyn and Bacon; 1993

33. Sattler JM. Assessment of Children's Intelligence. Revised and Updated. 3rd ed. San Diego, CA: Jerome Sattler; 1992.

34. Yu TY, Chen KL, Chou W, et al. Intelligence quotient discrepancy indicates levels of motor competence in preschool children at risk for developmental delays. Neuropsychiatr Dis Treat. 2016;12:501-510.

35. American Psychiatric Association. Diagnosis and Statistical Manual of Mental Disorder. Washington, DC: American Psychiatric Association Press; 2013.

36. Liao HF, Wang TM, Yao G, Lee WT. Concurrent validity of the Comprehensive Developmental Inventory for Infants and Toddlers with the Bayley Scales of Infant Development-II in preterm infants. J Formos Med Assoc. 2005;104(10):731-737.

37. Wang TM, Su CW, Liao HF, Lin LY, Chou KS, Lin SH. [The standardization of the Comprehensive Developmental Inventory for Infants and Toddlers]. Psychol Test. 1998;45:19-46. Chinese [with English abstract].

38. Hwang AW, Weng LJ, Liao HF. Construct validity of the Comprehensive Developmental Inventory for Infants and Toddlers. Pediatr Int. 2010;52(4):598-606.

39. Liao HF, Pan YL. Test-retest and inter-rater reliability for the Comprehensive Developmental Inventory for Infants and Toddlers diagnostic and screening tests. Early Hum Dev. 2005;81(11):927-937.

40. Liao HF, Yao G, Wang TM. Concurrent validity in Taiwan of the Comprehensive Developmental Inventory for Infants and Toddlers who were full-term infants. Percept Mot Skills. 2008;107(1):29-44.

41. Wang TM. [Predictive validity of Comprehensive Developmental Inventory for Infants and Toddlers (CDIIT)]. Bull Spec Educ. 2005;29:1-24. Chinese [with English abstract].
42. Wang TM, Liao HF. [Assessment accuracy and cut-off points of Comprehensive Developmental Inventory for Infants and Toddlers (CDIIT)]. Bull Spec Educ. 2007;32:1-15. Chinese [with English abstract].

43. Wu HY, Liao HF, Yao G, Lee WT, Wang TM, Hsieh JY. Diagnostic accuracy of the motor subtest of Comprehensive Developmental Inventory for Infants and Toddlers and the Peabody Developmental Motor Scales-Second Edition. J Formos Med Assoc. 2005;9:312-322.

44. Wechsler D. Manual for the Wechsler Preschool and Primary Scale of Intelligence-Revised (WPPSI). San Antonio, TX: Psychological Corporation; 1967.

45. Anastasi A. Psychological Testing. New York: Macmillan; 1988.

46. Seashore HG. Differences between verbal and performance IQs on the Wechsler Intelligence Scale for Children. J Consult Psychol. 1951;15(1):62-67.

47. Kaufman AS. Assessing Adolescent and Adult Intelligence. Needham, MA: Allyn and Bacon; 1990.

48. Field AP. Discovering Statistics using SPSS. London: Sage Publications Ltd; 2009.

49. PASW. PASW [Computer Software]. 18th ed. Chicago: SPSS; 2009.

50. Green D, Baird G, Barnett AL, Henderson L, Huber J, Henderson SE The severity and nature of motor impairment in Asperger's syndrome: a comparison with specific developmental disorder of motor function. $J$ Child Psychol Psychiatry. 2002;43(5):655-668.

51. Green D, Charman T, Pickles A, et al. Impairment in movement skills of children with autistic spectrum disorders. Dev Med Child Neurol. 2009;51(4):311-316.

52. Hilton C, Wente L, LaVesser P, Ito M, Reed C, Herzberg G. Relationship between motor skill impairment and severity in children with Asperger syndrome. Res Autism Spectr Disord. 2007;1(4):339-349

53. Provost B, Lopez BR, Heimerl S. A comparison of motor delays in young children: Autism spectrum disorder, developmental delay, and developmental concerns. J Autism Dev Disord. 2007;37(2):321-328.

54. Staples KL, Reid G. Fundamental movement skills and autism spectrum disorders. J Autism Dev Disord. 2010;40(2):209-217.
Neuropsychiatric Disease and Treatment

\section{Publish your work in this journal}

Neuropsychiatric Disease and Treatment is an international, peerreviewed journal of clinical therapeutics and pharmacology focusing on concise rapid reporting of clinical or pre-clinical studies on a range of neuropsychiatric and neurological disorders. This journal is indexed on PubMed Central, the 'PsycINFO' database and CAS,

\section{Dovepress}

and is the official journal of The International Neuropsychiatric Association (INA). The manuscript management system is completely online and includes a very quick and fair peer-review system, which is all easy to use. Visit http://www.dovepress.com/testimonials.php to read real quotes from published authors. 